\title{
A STABILITY ANALYSIS FOR FINITE VOLUME SCHEMES APPLIED TO THE MAXWELL SYSTEM
}

\author{
SOPHIE DEPEYRE ${ }^{1}$
}

\begin{abstract}
We present in this paper a stability study concerning finite volume schemes applied to the two-dimensional Maxwell system, using rectangular or triangular meshes. A stability condition is proved for the first-order upwind scheme on a rectangular mesh. Stability comparisons between the Yee scheme and the finite volume formulation are proposed. We also compare the stability domains obtained when considering the Maxwell system and the convection equation.

Résumé. Nous présentons dans cet article une étude de stabilité pour les schémas de type volumes finis appliqués au système de Maxwell bidimensionnel, sur des maillages en rectangles ou en triangles. Un résultat de stabilité est démontré pour le schéma décentré d'ordre un sur un maillage rectangulaire. Des comparaisons de stabilité entre le schéma de Yee et la formulation en volumes finis sont proposées. Nous comparons également les domaines de stabilité obtenus pour le système de Maxwell et pour l'équation d'advection.
\end{abstract}

AMS Subject Classification. 11C08, 11C20, 65C20, 65L06, 65M12, 65M60, 65T20.

Received: March 11, 1997.

\section{INTRODUCTION}

We are concerned, in this paper, with a stability study for finite volume schemes applied to the twodimensional time domain Maxwell system. This analysis is achieved for a homogeneous medium, for instance the vacuum.

We shall consider first-order and higher-order schemes on rectangular and triangular meshes. In the case of a first-order scheme using a rectangular mesh, a necessary and sufficient stability condition will be proved. This stability result will be compared to the one obtained for a Yee scheme [10], which is largely used in C.E.M. applications.

In order to increase the accuracy of finite-volume schemes as well as the stability domains, high-order schemes, both in time and space, are achieved by means of an upwinding parameter $\beta$ and a multi-step Runge-Kutta time discretization [4]. In this case, representations of the stability domains will be computed, in order to compare stability conditions for the Yee scheme and for third-order finite volume schemes. This stability analysis will allow us to draw the most efficient schemes, on triangular and rectangular meshes, from a class of high-order accurate methods.

\footnotetext{
Keywords and phrases. Finite volume, finite element, stability, Maxwell system, Fourier analysis.

1 CERMICS-INRIA, B.P. 93, 06902 Sophia-Antipolis Cedex, France.

Present address: Pôle Universitaire Léonard de Vinci, 92916 La Défense Cedex, France. e-mail: sophie-depeyre@devinci.fr
} 
A comparison of the stability limits obtained for the Maxwell system and for the scalar convection equation will also be proposed.

This paper is divided into three parts: in the two first ones, we recall the Maxwell equations and also the numerical approximation based on finite volumes; the third one is concerned with the stability study and the comparison of the stability results between the Yee scheme and finite-volume methods applied to the Maxwell system and to the convection equation.

\section{MAXWELL SYSTEM}

\subsection{Electromagnetic field equations}

The electric field $\mathbf{E}=\mathbf{E}(t, \mathbf{x})$ and the magnetic induction $\mathbf{B}=\mathbf{B}(t, \mathbf{x})$ are solutions in vacuum of the Maxwell equations:

$$
\left\{\begin{array}{l}
\frac{\partial \mathbf{E}}{\partial t}-c^{2} \operatorname{rot}(\mathbf{B})=-\frac{\mathbf{j}}{\epsilon_{\mathbf{0}}} \quad\left(\mathbf{x} \in \mathbb{R}^{3}, t>0\right) \\
\frac{\partial \mathbf{B}}{\partial t}+\operatorname{rot}(\mathbf{E})=0 \\
\operatorname{div}(\mathbf{E})=\frac{\rho}{\epsilon_{0}} \\
\operatorname{div}(\mathbf{B})=0
\end{array}\right.
$$

where $c$ is the light velocity, $\epsilon_{0}$ the vacuum electric permittivity and $\mu_{0}$ the vacuum magnetic permeability. These values satisfy the relation: $\epsilon_{0} \mu_{0} c^{2}=1$.

We denote by $\mathbf{j}=\mathbf{j}(t, \mathbf{x})$ and $\rho=\rho(t, \mathbf{x})$ the given current and the given charge densities which are related by the conservation law:

$$
\frac{\partial \rho}{\partial t}+\operatorname{div}(\mathbf{j})=0
$$

We assume that the initial electric field $\mathbf{E}_{0}$ and the magnetic induction $\mathbf{B}_{0}$ are such that:

$$
\operatorname{div} \mathbf{E}_{0}=\frac{\rho(t=0)}{\epsilon_{0}}=\frac{\rho_{0}}{\epsilon_{0}}, \quad \operatorname{div} \mathbf{B}_{0}=0
$$

One can easily prove that conditions (3) and the charge conservation law (2) imply that constraints $(1.3,1.4)$ are satisfied for all $t>0$. Hence, only the first two equations $(1.1,1.2)$ will be considered in the numerical model since $(1.3,1.4)$ are redundant in the continuous one.

\subsection{Conservative formulation and hyperbolic character}

System (1) can be written in the following conservative form:

$$
\mathbf{Q}_{t}+\mathbf{F}_{\mathbf{1}}(\mathbf{Q})_{x}+\mathbf{F}_{\mathbf{2}}(\mathbf{Q})_{y}+\mathbf{F}_{\mathbf{3}}(\mathbf{Q})_{z}=\mathbf{J}
$$

where

$$
\left\{\begin{array}{l}
\mathbf{Q}={ }^{t}\left(E_{1}, E_{2}, E_{3}, B_{1}, B_{2}, B_{3}\right) \\
\mathbf{F}_{\mathbf{1}}(\mathbf{Q})={ }^{t}\left(0, c^{2} B_{3},-c^{2} B_{2}, 0,-E_{3}, E_{2}\right) \\
\mathbf{F}_{\mathbf{2}}(\mathbf{Q})={ }^{t}\left(-c^{2} B_{3}, 0, c^{2} B_{1}, E_{3}, 0,-E_{1}\right) \\
\mathbf{F}_{\mathbf{3}}(\mathbf{Q})={ }^{t}\left(c^{2} B_{2},-c^{2} B_{1}, 0,-E_{2}, E_{1}, 0\right) \\
\mathbf{J}=-\frac{1}{\epsilon_{0}}{ }^{t}\left(j_{1}, j_{2}, j_{3}, 0,0,0\right)
\end{array}\right.
$$


or in condensed form:

$$
\mathbf{Q}_{t}+\vec{\nabla} \cdot \mathbb{F}(\mathbf{Q})=\mathbf{J}
$$

with $\mathbb{F}(\mathbf{Q})={ }^{t}\left(\mathbf{F}_{\mathbf{1}}(\mathbf{Q}) ; \mathbf{F}_{\mathbf{2}}(\mathbf{Q}) ; \mathbf{F}_{\mathbf{3}}(\mathbf{Q})\right)$.

One can easily check that system (5) is hyperbolic. Indeed, let us consider a linear combination of fluxes:

$$
\mathcal{F}(\mathbf{Q}, \boldsymbol{\eta})=\boldsymbol{\eta} \cdot \mathbb{F}(\mathbf{Q})
$$

where $\boldsymbol{\eta}={ }^{t}\left(\eta_{1}, \eta_{2}, \eta_{3}\right)$ is any nonzero vector of $\mathbb{R}^{3}$.

The Jacobian matrix $\mathcal{A}$ defined by:

$$
\mathcal{A}(\mathbf{Q}, \boldsymbol{\eta})=\boldsymbol{\eta} . \mathbb{F}^{\prime}(\mathbf{Q})=\eta_{1} \mathcal{A}_{1}+\eta_{2} \mathcal{A}_{2}+\eta_{3} \mathcal{A}_{3}, \quad\left(\mathcal{A}_{i}\right)_{i=1, \ldots, 3}=\frac{\partial}{\partial \mathbf{Q}} \mathbf{F}_{\mathbf{i}}(\mathbf{Q})
$$

is diagonalizable for any nonzero vector $\boldsymbol{\eta}$ of $\mathbb{R}^{3}$ and for any vector $\mathbf{Q}$ of $\mathbb{R}^{6}$.

Its three real eigenvalues of double multiplicity are given by:

$$
\left\{\begin{array}{l}
\lambda_{1}=c\|\boldsymbol{\eta}\| \\
\lambda_{2}=-c\|\boldsymbol{\eta}\| \\
\lambda_{3}=0 .
\end{array}\right.
$$

Two types of wave polarization are particularly interesting when modelling two dimensional problems : transverse electric polarizations noted $\mathbf{T E}\left(\mathbf{E} . \mathbf{e}_{\mathbf{z}}=0\right)$ and transverse magnetic polarizations noted $\mathbf{T M}\left(\mathbf{B} . \mathbf{e}_{\mathbf{z}}=0\right)$. Indeed, in the two-dimensional case, these polarizations allow the Maxwell system to be splitted according to these two types of waves. From now, we shall restrict our study to the transverse electric waves (TE). The TM case is treated similarly. In this case, (4) writes:

$$
\mathbf{Q}_{t}+\mathbf{F}_{\mathbf{1}}(\mathbf{Q})_{x}+\mathbf{F}_{\mathbf{2}}(\mathbf{Q})_{y}=\mathbf{J}
$$

where:

$$
\left\{\begin{array}{l}
\mathbf{Q}={ }^{t}\left(E_{1}, E_{2}, B_{3}\right) \\
\mathbf{F}_{\mathbf{1}}(\mathbf{Q})={ }^{t}\left(0, c^{2} B_{3}, E_{2}\right) \\
\mathbf{F}_{\mathbf{2}}(\mathbf{Q})={ }^{t}\left(-c^{2} B_{3}, 0,-E_{1}\right) \\
\mathbf{J}=-\frac{1}{\epsilon_{0}}{ }^{t}\left(j_{1}, j_{2}, 0\right) .
\end{array}\right.
$$

The conservative form as well as the hyperbolic character of the Maxwell system leads up naturally to the use of upwind schemes which are known to be well adapted to solve numerically hyperbolic conservative systems.

\section{Numerical approximation}

The two-dimensional time domain solver presented here is based on a finite volume formulation using structured triangular or rectangular meshes. We describe briefly in the following section the finite volume method applied to the Maxwell equations. For more details on this method, one may refer to [2-4].

\subsection{Spatial formulation}

Let $\mathcal{T}_{h}$ be a standard finite element discretization of $\Omega_{h}$, the polygonal approximation of a computational domain $\Omega$ :

$$
\Omega_{h}=\bigcup_{j=1}^{n t} T_{j}
$$




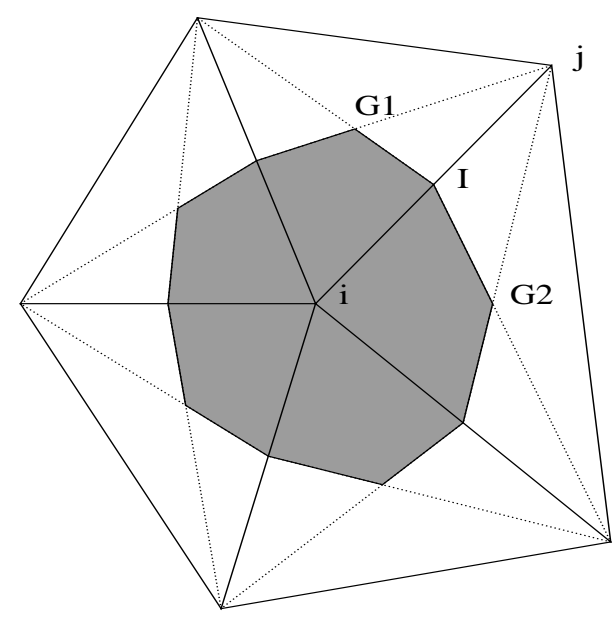

Figure 1. 2D cell $C_{i}$ for a triangular mesh.

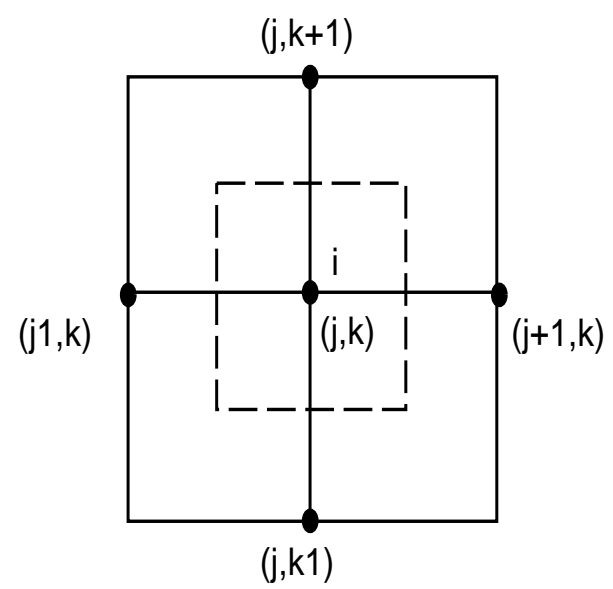

Figure 2. 2D cell $C_{i}$ for a rectangular mesh.

where $T_{j}$ is a triangular or a rectangular element and $n t$ is the number of elements. Another partition of $\Omega$ using finite volumes is then constructed as follows:

$$
\Omega=\bigcup_{i=1}^{n s} C_{i}
$$

where $n s$ is the number of nodes and $C_{i}$ is the control volume or cell whose construction is shown in Figures 1 and 2 .

A weak formulation is then obtained by integrating system (5) on each control volume $C_{i}$ taking the characteristic functions of the cells as test functions. 
Assuming partial derivative $\mathbf{Q}_{t}$ to be constant in space on $C_{i}$ and using a Green formula yields to the following equation written at each node of the mesh:

$$
\operatorname{Area}\left(C_{i}\right)\left(\mathbf{Q}_{t}\right)_{i}+\int_{\partial C_{i}} \mathbb{F}(\mathbf{Q}) \cdot \nu_{i} \mathrm{~d} \sigma=\int_{C_{i}} \mathbf{J} \mathrm{d} \mathbf{x}
$$

where $\boldsymbol{\nu}_{\boldsymbol{i}}$ is the unit normal exterior to $\partial C_{i}$.

The integral term in equation (7) is splitted into a sum of internal fluxes and boundary terms. Since we are mainly interested in the study of stability conditions, we shall consider periodic boundary conditions, which makes the contribution of these boundary terms to be zero.

$$
\operatorname{Area}\left(C_{i}\right)\left(\mathbf{Q}_{t}\right)_{i}+\sum_{j=1}^{N_{i}} \Phi_{i j}=\operatorname{Area}\left(C_{i}\right) \mathbf{J}_{\mathbf{i}}
$$

where $N_{i}$ is the number of the neighbours of the node $i$ and $\Phi_{i j}$ is an approximation of the internal flux $\int_{\partial C_{i} \cap \partial C_{j}} \mathbb{F}(\mathbf{Q}) \cdot \nu_{i j} \mathrm{~d} \sigma$ which will be discussed in the sequel.

\subsection{First-order upwind scheme}

Since the Maxwell system is hyperbolic, we choose an upwind approximation for the evaluation of the numerical fluxes $\Phi_{i j}$. Let us set:

$$
\boldsymbol{\eta}=\int_{\partial C_{i} \cap \partial C_{j}} \boldsymbol{\nu}_{\boldsymbol{i j}} \mathrm{d} \sigma
$$

where $\partial C_{i} \cap \partial C_{j}$ represents the common interface between the two cells $C_{i}$ and $C_{j}$. We recall that the Maxwell equations in vacuum form is a linear system with constant coefficients. Thus all first-order upwind schemes reduce to the classical I.C.R. (Isaac-Courant-Reeves) scheme [7] which writes:

$$
\Phi_{i j}=\Phi\left(\mathbf{Q}_{i}, \mathbf{Q}_{j}, \boldsymbol{\eta}\right)=\frac{\mathcal{F}\left(\mathbf{Q}_{i}, \boldsymbol{\eta}\right)+\mathcal{F}\left(\mathbf{Q}_{j}, \boldsymbol{\eta}\right)}{2}-\frac{1}{2}|\mathcal{A}(\boldsymbol{\eta})|\left(\mathbf{Q}_{j}-\mathbf{Q}_{i}\right)
$$

where $\mathbf{Q}_{i}$ denotes the value of $\mathbf{Q}$ at node $i$ and $\mathcal{A}(\boldsymbol{\eta})$ is the Jacobian matrix of $\mathcal{F}(\mathbf{Q}, \boldsymbol{\eta})$.

\subsection{High order approximation}

The MUSCL (Monotonic Upwind Schemes for Conservation Laws) method [5,8] allows us to increase the precision of the schemes by defining new values $\mathbf{Q}_{i j}$ and $\mathbf{Q}_{j i}$ at the interface of the cells without altering the numerical fluxes function $\Phi$. In the MUSCL method, these values are obtained by using a linear interpolation on each cell. We choose here a $\beta$-scheme formulation which writes:

$$
\left\{\begin{array}{l}
\boldsymbol{\Phi}_{\boldsymbol{i j}}=\mathbf{\Phi}_{\boldsymbol{i j}}\left(\mathbf{Q}_{i j}, \mathbf{Q}_{j i}\right) \\
\mathbf{Q}_{i j}=\mathbf{Q}_{i}+\frac{1}{2}\left\{(1-2 \beta)\left(\mathbf{Q}_{j}-\mathbf{Q}_{i}\right)+2 \beta \vec{\nabla} \mathbf{Q}_{i}^{H} \cdot \mathbf{S}_{i} \mathbf{S}_{j}\right\} \\
\mathbf{Q}_{j i}=\mathbf{Q}_{j}-\frac{1}{2}\left\{(1-2 \beta)\left(\mathbf{Q}_{j}-\mathbf{Q}_{i}\right)+2 \beta \vec{\nabla} \mathbf{Q}_{j}^{H} \cdot \mathbf{S}_{i} \mathbf{S}_{j}\right\}
\end{array}\right.
$$

where $\beta$ is an upwinding parameter whose value determines the accuracy of the scheme. Choosing $\beta=1 / 3$ gives a third-order accurate scheme in space for structured meshes [3,4]. The formulation requires the evaluation of a nodal gradient $(\vec{\nabla} \mathbf{Q})_{i, j}^{H}$ which can be defined in several ways. We use here a finite element approach. 
In the case of a rectangular mesh, it writes:

$$
\begin{aligned}
\vec{\nabla} \mathbf{Q}_{i}^{H}{ }_{R} & =\frac{1}{\operatorname{Area}\left(\operatorname{Supp}\left(\varphi_{i}\right)\right)} \int_{\operatorname{Supp}\left(\varphi_{i}\right)} \vec{\nabla} \mathbf{Q} \mathrm{d} \mathbf{x} \\
& =\frac{1}{\operatorname{Area}\left(\operatorname{Supp}\left(\varphi_{i}\right)\right)} \sum_{R, i \in R} \sum_{k=1}^{4} \mathbf{Q}_{i^{k}} \int_{R} \vec{\nabla} \varphi_{i^{k}} \mathrm{~d} \mathbf{x}
\end{aligned}
$$

where the $i^{k}(k=1, \ldots, 4)$ are the four vertices of the rectangle $R$ and $\vec{\nabla} \varphi_{i^{k}}$ is the gradient of the bilinear $Q^{1}$ function at node $i^{k}$.

In the case of a triangular mesh, we use the following definition:

$$
\begin{aligned}
\vec{\nabla} \mathbf{Q}_{i}^{H} T & =\frac{1}{\operatorname{Area}\left(\operatorname{Supp}\left(\varphi_{i}\right)\right)} \int_{\operatorname{Supp}\left(\varphi_{i}\right)} \vec{\nabla} \mathbf{Q} \mathrm{d} \mathbf{x} \\
& =\frac{1}{\operatorname{Area}\left(C_{i}\right)} \sum_{T, i \in T} \frac{\operatorname{Area}(T)}{3} \sum_{k=1}^{4} \mathbf{Q}_{i^{k}} \vec{\nabla} \varphi_{i^{k}}(T)
\end{aligned}
$$

where the $i^{k}(k=1,2,3)$ are the three vertices of the triangle $T$ and $\vec{\nabla} \varphi_{i^{k}}(T)$ is the gradient of the linear $P^{1}$ function at node $i^{k}$, which is constant on $T$.

\subsection{Time integration}

The time accuracy for unsteady problems is important that is why we choose explicit accurate time schemes. We use a Runge-Kutta multi-step explicit method; the step number for the accuracy of the scheme is fixed with regard to the value of $\beta$. The $R K r$ algorithm is given below (in our case $r=1, \ldots, 3$ ):

$$
\left\{\begin{aligned}
\mathbf{Q}^{0} & =\mathbf{Q}^{n} \\
\mathbf{Q}^{l} & =\mathbf{Q}^{0}-\frac{\Delta t}{(r+1-l)} \Phi\left(\mathbf{Q}^{l-1}\right) \quad l=1, \ldots, r \\
\mathbf{Q}^{n+1} & =\mathbf{Q}^{r}
\end{aligned}\right.
$$

where $t^{n}=n \Delta t$ and $\Phi\left(\mathbf{Q}^{l-1}\right)$ represent the fluxes calculated with fields $\mathbf{Q}^{l-1}$. For the values $\beta=1 / 3$ and $r=3$, the scheme is third-order accurate in time and space since the Maxwell system is linear.

\section{Stability ANALYSIS}

We study here the stability of the schemes presented above for both rectangular and triangular meshes. The Maxwell system is written dimensionless and we choose $c=1$. We consider the first-order accurate scheme and we present a proof of the stability condition in the case of a rectangular grid. Then we study the $\beta$-scheme stability on both rectangular and triangular meshes by adjusting the parameter $\beta$. Stability study is based on Von Neumann analysis [1], but we first introduce some definitions before developing this analysis. We note:

$$
Q_{j, k}^{n}=\hat{Q}^{n} e^{i\left(j \theta_{1}+k \theta_{2}\right)} \quad(p, q) \in \mathbb{Z}^{2}
$$

where $i^{2}=-1$. Then we obtain the relation:

$$
\hat{Q}^{n+1}=G_{\theta_{1}, \theta_{2}} \hat{Q}^{n}
$$


where $G_{\theta_{1}, \theta_{2}}$ is the $3 \times 3$ amplification matrix of the scheme which depends on the time increment $\Delta t$ and the Fourier angles $\theta_{1}, \theta_{2}$.

\subsection{First-order accurate schemes}

We recall that a necessary and sufficient stability condition (Von Neumann condition) writes:

$$
\forall\left(\theta_{1}, \theta_{2}\right) \in[0,2 \pi]^{2}, \quad r\left(G_{\theta_{1}, \theta_{2}}\right)=\max _{l=1,2,3}\left|\mu_{\theta_{1}, \theta_{2}}^{l}\right| \leq 1
$$

where $\mu_{\theta_{1}, \theta_{2}}^{l}$ are the eigenvalues of $G_{\theta_{1}, \theta_{2}}$ and $r$ is the spectral radius of the matrix $G_{\theta_{1}, \theta_{2}}$.

\subsubsection{Rectangular mesh}

In this part, we shall establish a necessary and sufficient stability condition for the first-order finite volume scheme on a rectangular mesh. The amplification matrix $G_{\theta_{1}, \theta_{2}}$ writes in this case:

$$
G_{\theta_{1}, \theta_{2}}=\operatorname{Id}-\Delta t\left(\begin{array}{ccc}
X_{2} & 0 & \frac{i}{\Delta y} \sin \left(\theta_{2}\right) \\
0 & X_{1} & -\frac{i}{\Delta x} \sin \left(\theta_{1}\right) \\
\frac{i}{\Delta y} \sin \left(\theta_{2}\right)-\frac{i}{\Delta x} \sin \left(\theta_{1}\right) & X_{1}+X_{2}
\end{array}\right)
$$

where $X_{1}=\frac{2}{\Delta x} \sin ^{2} \frac{\theta_{1}}{2}, X_{2}=\frac{2}{\Delta y} \sin ^{2} \frac{\theta_{2}}{2}$.

We notice that $G_{\theta_{1}, \theta_{2}}$ is a complex symmetric matrix.

Theorem 4.1. The first-order finite volume scheme applied to the Maxwell system using a rectangular mesh is stable if and only if $\frac{\Delta t}{\Delta x}+\frac{\Delta t}{\Delta y} \leq 1$.

Proof. We prove first that the condition $\frac{\Delta t}{\Delta x}+\frac{\Delta t}{\Delta y} \leq 1$ is necessary and then that it is sufficient.

Proposition 4.1. If the scheme is stable then $\frac{\Delta t}{\Delta x}+\frac{\Delta t}{\Delta y} \leq 1$.

Demonstrating this assertion is equivalent to show that if $\frac{\Delta t}{\Delta x}+\frac{\Delta t}{\Delta y}>1$ there exits a couple $\left(\theta_{1}, \theta_{2}\right)$ for which $\max _{l=1,2,3}\left|\mu_{\theta_{1}, \theta_{2}}^{l}\right|>1$. Taking $\left(\theta_{1}, \theta_{2}\right)=(\pi, \pi)$ leads up to:

$$
G_{\theta_{1}, \theta_{2}}=\operatorname{Id}-\Delta t\left(\begin{array}{ccc}
\frac{2}{\Delta y} & 0 & 0 \\
0 & \frac{2}{\Delta x} & 0 \\
0 & 0 & \frac{2}{\Delta x}+\frac{2}{\Delta y}
\end{array}\right)
$$

and $r\left(G_{\theta_{1}, \theta_{2}}\right)=\left|1-2\left(\frac{\Delta t}{\Delta x}+\frac{\Delta t}{\Delta y}\right)\right|$.

Hence taking $\frac{\Delta t}{\Delta x}+\frac{\Delta t}{\Delta y}>1$ leads clearly to an unstable scheme, which ends the proof of proposition (4.1). 
Proposition 4.2. If $\frac{\Delta t}{\Delta x}+\frac{\Delta t}{\Delta y} \leq 1$, then the scheme is stable.

Proof. We first define a new matrix $H_{\theta_{1}, \theta_{2}}$ by multiplying the third column of $G_{\theta_{1}, \theta_{2}}$ by $-i$ and the third line by $i$. One can easily check that $G_{\theta_{1}, \theta_{2}}$ and $H_{\theta_{1}, \theta_{2}}$ are similar. Hence they have the same eigenvalues, and the stability condition (12) is identical when considering $H_{\theta_{1}, \theta_{2}}$ as the scheme amplification matrix. $H_{\theta_{1}, \theta_{2}}$ presents the advantage to be real and can be splitted into: $H_{\theta_{1}, \theta_{2}}=\mathrm{Id}-\Delta t\left(D_{\theta_{1}, \theta_{2}}+A_{\theta_{1}, \theta_{2}}\right)$ where $D_{\theta_{1}, \theta_{2}}$ is a real diagonal matrix and $A_{\theta_{1}, \theta_{2}}$ is a real antisymmetric one.

$$
D_{\theta_{1}, \theta_{2}}=\left(\begin{array}{ccc}
X_{2} & 0 & 0 \\
0 & X_{1} & 0 \\
0 & 0 & X_{1}+X_{2}
\end{array}\right), \quad A_{\theta_{1}, \theta_{2}}=\left(\begin{array}{ccc}
0 & 0 & -\frac{1}{\Delta y} \sin \theta_{2} \\
0 & 0 & \frac{1}{\Delta x} \sin \theta_{1} \\
\frac{1}{\Delta y} \sin \theta_{2}-\frac{1}{\Delta x} \sin \theta_{1} & 0
\end{array}\right) .
$$

The matrix $\left(D_{\theta_{1}, \theta_{2}}+A_{\theta_{1}, \theta_{2}}\right)$ has either three real eigenvalues or one real eigenvalue and two complex conjugate ones. Concerning the real eigenvalues we have the following result:

Lemma 4.1. The real eigenvalues $\lambda_{r, \theta_{1}, \theta_{2}}$ of $\left(D_{\theta_{1}, \theta_{2}}+A_{\theta_{1}, \theta_{2}}\right)$ verify

$$
0 \leq \min \left(X_{1}, X_{2}\right) \leq \lambda_{r, \theta_{1}, \theta_{2}} \leq X_{1}+X_{2} .
$$

We omit from now the subscripts $\theta_{1}, \theta_{2}$ in what follows. This lemma and all the following ones will be proved further.

Let $\mu$ be the eigenvalues of $H$ and $\lambda$ the eigenvalues of $(D+A)$. Then we have $\mu=1-\Delta t \lambda$.

We first consider the case of the real eigenvalues $\mu_{r}$ of $H$.

Equation (12) implies: $\left|\mu_{r}\right| \leq 1 \forall\left(\theta_{1}, \theta_{2}\right)$ i.e. $-1 \leq 1-\Delta t \lambda_{r} \leq 1 \forall\left(\theta_{1}, \theta_{2}\right)$.

Using Lemma 4.1 one has $0 \leq \lambda_{r} \leq X_{1}+X_{2}$, furthermore $X_{1}+X_{2} \leq \frac{2}{\Delta x}+\frac{2}{\Delta y}$. Therefore, if the condition $\frac{\Delta t}{\Delta x}+\frac{\Delta t}{\Delta y} \leq 1$ is assumed one obtains $0 \leq \Delta t \lambda_{r} \leq 2$ and then $\left|\mu_{r}\right| \leq 1$.

We now consider the case of the complex eigenvalues $\mu_{c}$ of $H$. First we have:

$$
\left|\mu_{c}\right|^{2}=1-2 \Delta t \operatorname{Re}\left(\lambda_{c}\right)+\Delta t^{2}\left|\lambda_{c}\right|^{2} .
$$

The condition $\left|\mu_{c}\right| \leq 1$ writes $\Delta t^{2}\left|\lambda_{c}\right|^{2}-2 \Delta t \operatorname{Re}\left(\lambda_{c}\right) \leq 0$.

Assuming the real eigenvalue $\lambda_{r}$ is strictly positive, we obtain by multiplying the previous inequality by $\lambda_{r}$ :

$$
\Delta t^{2} \lambda_{r}\left|\lambda_{c}\right|^{2}-2 \Delta t \lambda_{r} \operatorname{Re}\left(\lambda_{c}\right) \leq 0 .
$$

Furthermore one has:

$\lambda_{r}\left|\lambda_{c}\right|^{2}=\operatorname{det}(D+A)=2 X_{1} X_{2}\left(\frac{1}{\Delta x}+\frac{1}{\Delta y}\right), \lambda_{r}+2 \operatorname{Re}\left(\lambda_{c}\right)=\operatorname{Tr}(D+A)=2\left(X_{1}+X_{2}\right)$.

Inequality (14) transforms into:

$$
P\left(\lambda_{r}\right)=\lambda_{r}^{2}-2\left(X_{1}+X_{2}\right) \lambda_{r}+2 X_{1} X_{2}\left(\frac{\Delta t}{\Delta x}+\frac{\Delta t}{\Delta y}\right) \leq 0 .
$$

Lemma 4.2. If the condition $\frac{\Delta t}{\Delta x}+\frac{\Delta t}{\Delta y} \leq 1$ is achieved, then $P\left(\lambda_{r}\right) \leq 0$.

Using Lemma 4.2 allows us to conclude that $\left|\mu_{c}\right| \leq 1 \forall\left(\theta_{1}, \theta_{2}\right)$.

The following Lemma treats the case $\lambda_{r}=0$. 
Lemma 4.3. If $\frac{\Delta t}{\Delta x}+\frac{\Delta t}{\Delta y} \leq 1$, the condition $\left|\mu_{c}\right| \leq 1 \forall\left(\theta_{1}, \theta_{2}\right)$ is ensured.

We have finally proved that if $\frac{\Delta t}{\Delta x}+\frac{\Delta t}{\Delta y} \leq 1$, then the scheme is stable which concludes the proof of Proposition 4.2 and the demonstration of Theorem 4.1.

We establish in the sequel the proof of all the intermediary lemma.

Proof of Lemma 4.1. Let $\mathbf{v}$ be the eigenvector of the real matrix $(D+A)$ associated to the eigenvalue $\lambda_{r}$. We have: $(D+A) \mathbf{v}=\lambda_{r} \mathbf{v}$ and ${ }^{t} \mathbf{v}(D+A) \mathbf{v}={ }^{t} \mathbf{v} \lambda_{r} \mathbf{v}$. As $A$ is an antisymmetric matrix, from ${ }^{t} \mathbf{v} A \mathbf{v}=0$ we deduce ${ }^{t} \mathbf{v} D \mathbf{v}={ }^{t} \mathbf{v} \lambda_{r} \mathbf{v}$ which writes:

$$
\left(X_{2}-\lambda_{r}\right) v_{1}^{2}+\left(X_{1}-\lambda_{r}\right) v_{2}^{2}+\left(X_{1}+X_{2}-\lambda_{r}\right) v_{3}^{2}=0
$$

where $v_{i}(i=1,2,3)$ are the components of the eigenvector $\mathbf{v}$.

We note that $X_{1}+X_{2}-\lambda_{r} \geq \max \left(X_{1}-\lambda_{r}, X_{2}-\lambda_{r}\right)$ as $X_{1}$ and $X_{2}$ are positive.

Since the coefficients in front of $v_{i}$ can not have all the same sign, one can deduce that $X_{1}+X_{2}-\lambda_{r}>0$ and $\left(X_{1}-\lambda_{r}\right)$ or $\left(X_{2}-\lambda_{r}\right)$ are negative. Thus one can conclude that $\lambda_{r} \leq X_{1}+X_{2}$ and $\lambda_{r} \geq \min \left(X_{1}, X_{2}\right) \geq 0$.

Proof of Lemma 4.2. We recall that:

$$
P\left(\lambda_{r}\right)=\lambda_{r}^{2}-2\left(X_{1}+X_{2}\right) \lambda_{r}+2 X_{1} X_{2}\left(\frac{\Delta t}{\Delta x}+\frac{\Delta t}{\Delta y}\right)
$$

The discriminant of $P$ writes:

$$
\Delta=4\left(X_{1}+X_{2}\right)^{2}-8 X_{1} X_{2}\left(\frac{\Delta t}{\Delta x}+\frac{\Delta t}{\Delta y}\right)
$$

Assuming the condition $\frac{\Delta t}{\Delta x}+\frac{\Delta t}{\Delta y} \leq 1$ leads to $\Delta \geq 4\left(X_{1}^{2}+X_{2}^{2}\right) \geq 0$.

The particular case $\Delta=0$ corresponds to $X_{1}^{2}+X_{2}^{2}=0$ which is equivalent to $\lambda_{r}=0$ in view of Lemma 4.1. Since we consider the case $\lambda_{r} \neq 0$, the discriminant is strictly positive and the polynomial $P\left(\lambda_{r}\right)$ has two distinct roots $r_{1}, r_{2}$ given by:

$$
r_{1}=\frac{2\left(X_{1}+X_{2}\right)-\sqrt{\Delta}}{2}, r_{2}=\frac{2\left(X_{1}+X_{2}\right)+\sqrt{\Delta}}{2}
$$

We obtain that $P\left(\lambda_{r}\right) \leq 0$ ever since $\lambda_{r} \in\left[r_{1}, r_{2}\right]$. Lemma 4.1 establishes that $0<\lambda_{r} \leq X_{1}+X_{2} \leq r_{2}$, then we still have to show that $\lambda_{r} \geq r_{1}$.

From $\Delta \geq 4\left(X_{1}^{2}+X_{2}^{2}\right)$ we have $r_{1} \leq X_{1}+X_{2}-\sqrt{X_{1}^{2}+X_{2}^{2}}$. Furthermore $\sqrt{X_{1}^{2}+X_{2}^{2}} \geq \max \left(X_{1}, X_{2}\right)$ and $X_{1}+X_{2}-\sqrt{X_{1}^{2}+X_{2}^{2}} \leq X_{1}+X_{2}-\max \left(X_{1}, X_{2}\right)=\min \left(X_{1}, X_{2}\right) \leq \lambda_{r}$ thanks to Lemma 4.1.

Finally $r_{1} \leq \lambda_{r} \leq r_{2}$ and $P\left(\lambda_{r}\right) \leq 0$ which ends the proof of Lemma 4.2.

Proof of Lemma 4.3. We consider here the case of a zero eigenvalue $\lambda_{r}$. From Lemma 4.1, if $\lambda_{r}=0$ then $\min \left(X_{1}, X_{2}\right)=0$ that is to say $\theta_{1}=2 k \pi$ or $\theta_{2}=2 k \pi,(k \in \mathbb{Z})$. Conversely, if $\theta_{1}$ or $\theta_{2}=2 k \pi$ then $\lambda_{r}=0$. Hence $\lambda_{r}=0$ is equivalent to $\theta_{1}=2 k \pi$ or $\theta_{2}=2 k \pi$. 


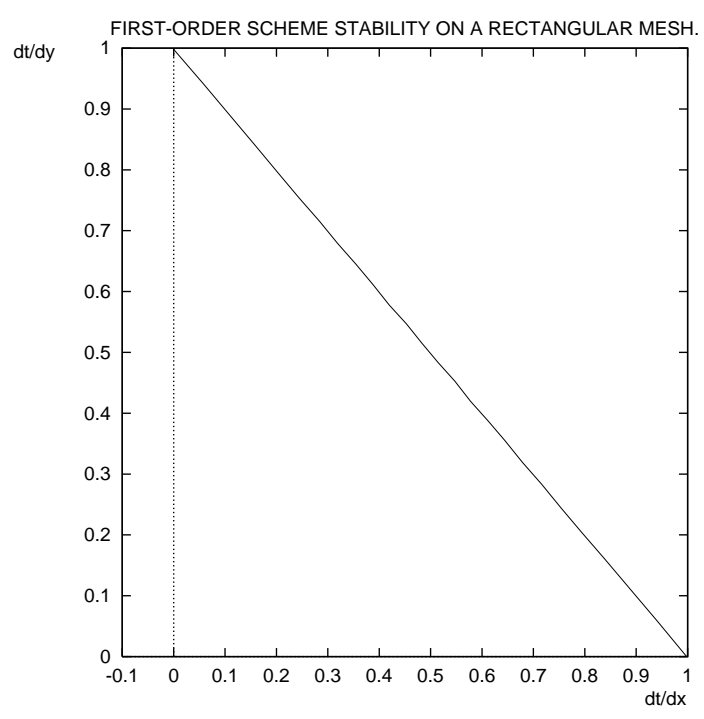

Figure 3. Maxwell system and convection equation.

Assuming $\theta_{1}=2 k \pi$, the matrix $(D+A)$ writes:

$$
D+A=\left(\begin{array}{ccc}
X_{2} & 0-\frac{1}{\Delta y} \sin \theta_{2} \\
0 & 0 & 0 \\
\frac{1}{\Delta y} \sin \theta_{2} & 0 & X_{2}
\end{array}\right)
$$

In this case, the eigenvalues of the matrix are: $0, X_{2} \pm \frac{i}{\Delta y} \sin \theta_{2}$. The condition $\left|\mu_{c}\right|^{2}=\left|1-\Delta t \lambda_{c}\right|^{2} \leq 1$ writes: $\Delta t^{2} X_{2}^{2}+\frac{\Delta t^{2}}{\Delta y^{2}} \sin ^{2} \theta_{2}-2 \Delta t X_{2} \leq 0$

One can easily check that this condition is achieved since $\frac{\Delta t}{\Delta y} \leq 1$. In the same way if we consider the case $\theta_{2}=2 k \pi$, the stability condition is satisfied if $\frac{\Delta t}{\Delta x} \leq 1$.

To sum up in the case of a zero eigenvalue, the condition $\frac{\Delta t}{\Delta x}+\frac{\Delta t}{\Delta y} \leq 1$ implies $\left|\mu_{c}\right| \leq 1$, which ends the proof of Lemma 4.3 .

We have proved that a necessary and sufficient stability condition for the first-order scheme on a rectangular mesh was $\frac{\Delta t}{\Delta x}+\frac{\Delta t}{\Delta y} \leq 1$

A way to represent the stability domain is to obtain numerically the maximum values of the couple $\left(\frac{\Delta t}{\Delta x}, \frac{\Delta t}{\Delta y}\right)$ such that the condition (12) may be verified. To represent this domain, we choose the variables $\frac{\Delta t}{\Delta x}$ and $\frac{\Delta t}{\Delta y}$ as coordinates in the plane. 


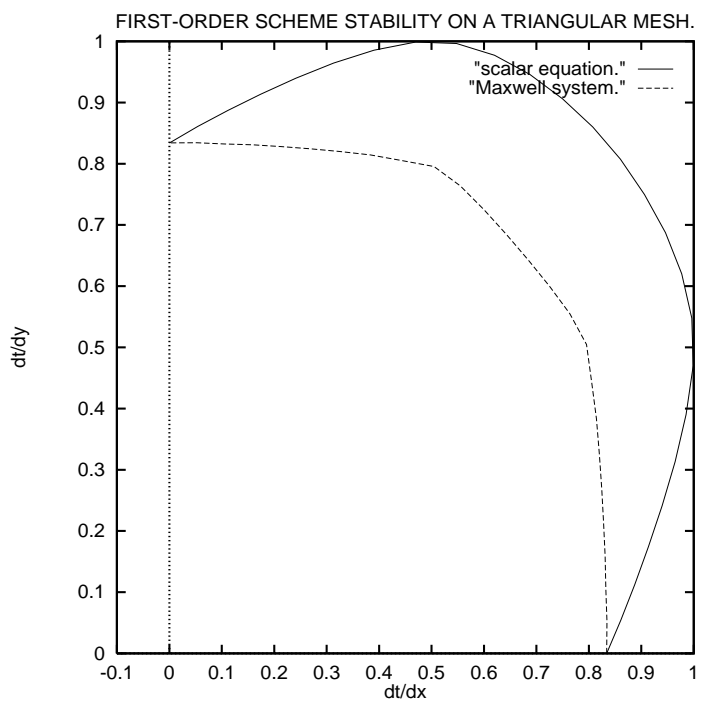

FiguRE 4. Maxwell system and convection equation.

\section{Remarks.}

- First we can notice that if we consider one direction infinite, for instance $\Delta y$, we obtain the monodimensional stability condition $\frac{\Delta t}{\Delta x} \leq 1$. This stability condition is the most restrictive when we choose $\Delta x=\Delta y$.

We note also that the stability domain represented on Figure 3 is the same as the one obtained when considering the first-order scheme applied to the two-dimensional scalar convection equation $u_{t}+u_{x}+u_{y}=0$ on a rectangular mesh. For more details on the stability analysis concerning the convection equation, one may refer to $[4,6]$.

- Maxwell system $(1.1,1.2)$ can be written into a non conservative form:

$$
\mathbf{Q}_{t}+A \mathbf{Q}_{x}+B \mathbf{Q}_{y}=0
$$

where $\mathrm{A}$ and $\mathrm{B}$ are the Jacobians of the fluxes $\mathbf{F}_{\mathbf{1}}(\mathbf{Q})$ and $\mathbf{F}_{\mathbf{2}}(\mathbf{Q})$.

In the monodimensional case, one can diagonalize the Jacobian matrix which leads up to a splitted system: each component is solution of the convection equation with speeds $(\mathrm{c},-\mathrm{c}, 0)$.

Unfortunately, in the two-dimensional case, the matrixes A and B are not diagonalizable in the same basis for the two space coordinates $(x, y)$. Thus it is not possible to transform the Maxwell system in order to obtain a system which each component may verify the convection equation, as it is in one dimension. However we find the same stability condition for the first-order scheme applied either to the Maxwell system or to the convection equation. As we shall see later, we do not observe the same concerning the $\beta$-schemes and the schemes using a triangular mesh.

\subsubsection{Triangular mesh}

In this part we study the stability in the case of a first-order scheme applied to the Maxwell system. The mesh used here is a structured triangular mesh obtained by cutting rectangles diagonally. We use again a Fourier analysis but in this case the matrix $H$ writes: $H=\operatorname{Id}-\Delta t(D+A+S)$ where $S$ is a symmetric matrix, which prevents us to apply the same demonstration as for the rectangular mesh. 


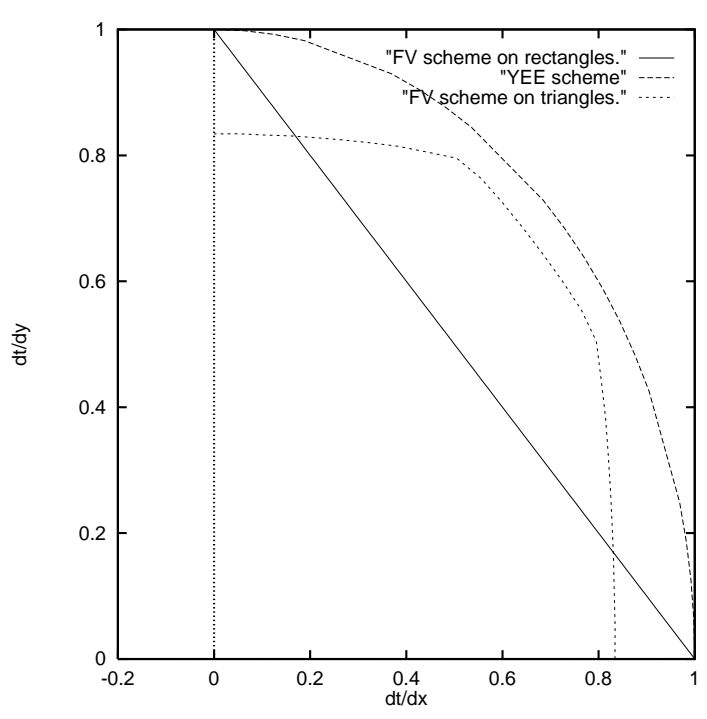

Figure 5. Stability domains for Yee and first-order finite volume schemes.

The eigenvalues of the amplification matrix are calculated numerically in order to obtain numerically a sufficient stability condition verifying (12). As done in the rectangular case, we choose the variables $\left(\frac{\Delta t}{\Delta x}, \frac{\Delta t}{\Delta y}\right)$ to represent the stability domain. A comparison between the convection equation and the Maxwell system is shown in Figure 4. We notice that the stability domain obtained when considering the convection equation is wider than the one obtained with the Maxwell system.

If we choose $\Delta x=\Delta y$, we can take $\mathrm{CFL}=1.17$ in the case of the convection equation, and $\mathrm{CFL}=0.93$ for the Maxwell system, where CFL is the Courant-Friedricks-Levy number. The stability limit is generally higher for the triangular mesh (see Figs. 3 and 4 ).

However, when a direction $\Delta x$ or $\Delta y$ is almost infinite, then the rectangular mesh gives a higher stability limit.

\subsubsection{Comparison with the Yee scheme}

One recalls that Yee introduced a set of finite-difference equations to discretize Maxwell equations. Yee algorithm consists in using finite difference expressions for the space and time derivatives, and in positioning the components of $\mathbf{E}$ and $\mathbf{B}$ orthogonally to each other. In order to achieve a second-order accurate scheme, in time and space, $\mathbf{E}$ and $\mathbf{B}$ are evaluated at half-time and half-space steps. The stability criterion for the two-dimensional Yee scheme writes as:

$$
\Delta t \sqrt{\frac{1}{\Delta x^{2}}+\frac{1}{\Delta y^{2}}} \leq 1
$$

and a proof of the above result can be found in [9].

Choosing the variables $\frac{\Delta t}{\Delta x}$ and $\frac{\Delta t}{\Delta y}$ to represent the stability domain leads up to a quarter-circular unit domain. Therefore, this stability condition is less restrictive than the one obtained for the first-order upwind scheme on both rectangular and triangular meshes (see Fig. 5). It is mainly due to the second-order accuracy of the Yee scheme. 


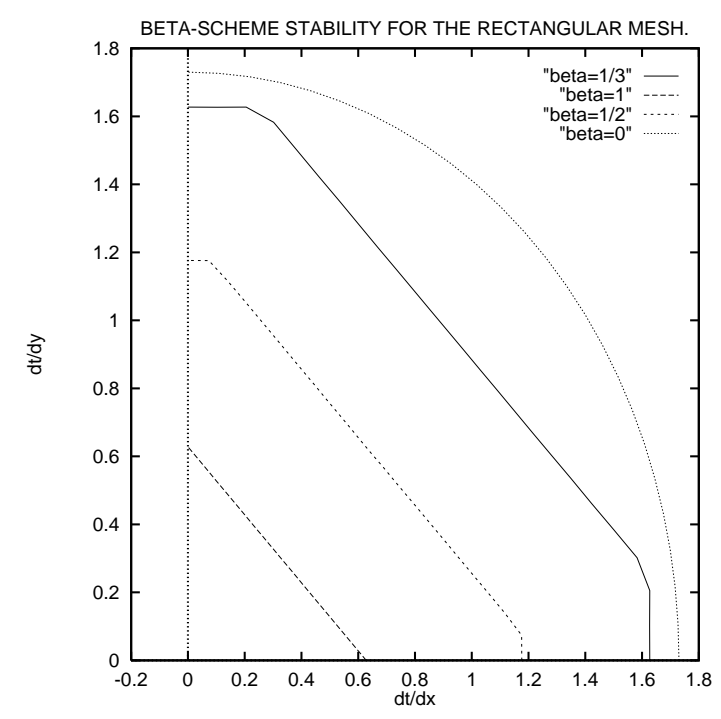

FiguRE 6. Maxwell system.

\subsection{Higher order schemes}

In the case of a three-step Runge-Kutta time integration we introduce the characteristic polynomial

$$
g(z)=1+z+\frac{z^{2}}{2}+\frac{z^{3}}{6}
$$

For $z=A \Delta t$, we recall that the polynomial $\mathcal{G}(A \Delta t)$ represents the amplification matrix of the Runge-Kutta method applied to the differential system $Q_{t}=A Q$ where A is the $3 \times 3$ scheme matrix. We obtain the following relation using a Fourier analysis:

$$
\hat{Q}^{n+1}=\mathcal{G}_{\theta_{1}, \theta_{2}}(A \Delta t) \hat{Q}^{n}
$$

and Von Neumann theorem (12) still applies to $\mathcal{G}_{\theta_{1}, \theta_{2}}$.

\subsubsection{Rectangular mesh}

In this section we plot some stability domains computed with different values of the upwinding parameter $\beta$.

We recall that for $\beta=0$ we obtain a centered scheme, for $\beta=1 / 2$ the scheme is half-centered, $\beta=1$ gives an upwind scheme.

Figure 6 shows that the closer to $1 \beta$ is, the smaller the stability limit is, which means that using a centered scheme allows us to take a higher time step.

As a comparison, we represent in Figure 7 the stability domain in the case of the convection equation. Although they vary in the same way with $\beta$, the stability domains are different except for $\beta=1$ where we obtain in both cases the numerical stability limit: $\frac{\Delta t}{\Delta x}+\frac{\Delta t}{\Delta y}=0.62$. If we choose $\Delta x$ or $\Delta y$ infinite we find in both cases the monodimensional stability limit. For $\Delta x$ and $\Delta y$ finite and for a fixed value of $\beta$ the stability domain is wider for the Maxwell system than for the advection equation, especially for $\beta=1 / 3$ or $\beta=0$.

\subsubsection{Triangular mesh}

The stability domains obtained for the $\beta$-schemes on a triangular mesh are still different concerning the Maxwell system and the convection equation (see Figs. 8 and 9). As for the rectangular case, the stability limit 


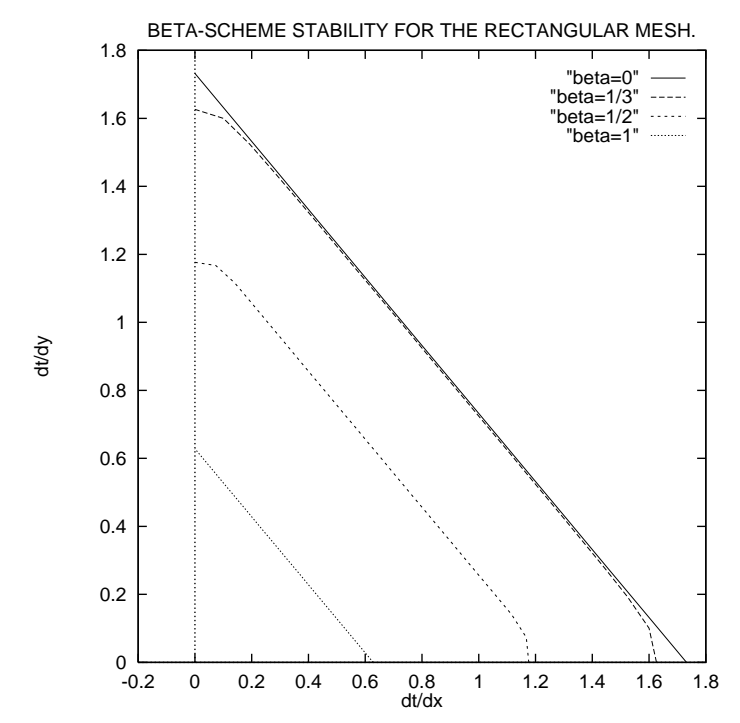

Figure 7. Convection equation.

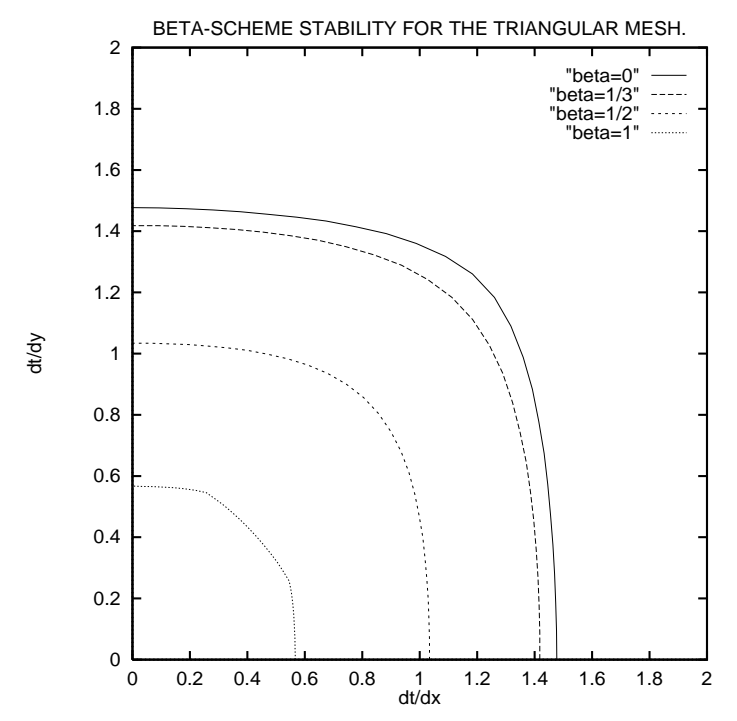

FiguRE 8. Maxwell system.

decreases when $\beta$ is closer to 1 . However, the stability domains are wider in the case of the convection equation for all value of the upwinding parameter $\beta$.

If we take $\Delta x$ or $\Delta y$ infinite we find again the same monodimensional stability limit for the Maxwell system and for the convection equation.

We can notice that using a triangular mesh gives the privilege to the direction $\Delta x=\Delta y$ concerning the stability: it is the direction where we can choose the highest time step, on the contrary to the rectangular mesh where imposing $\Delta x=\Delta y$ is the most restrictive choice. However, if a direction $\Delta x$ or $\Delta y$ is close to infinity, the use of rectangular meshes is more interesting concerning the stability limit. The two last remarks concern the Maxwell system as well as the scalar convection equation. 


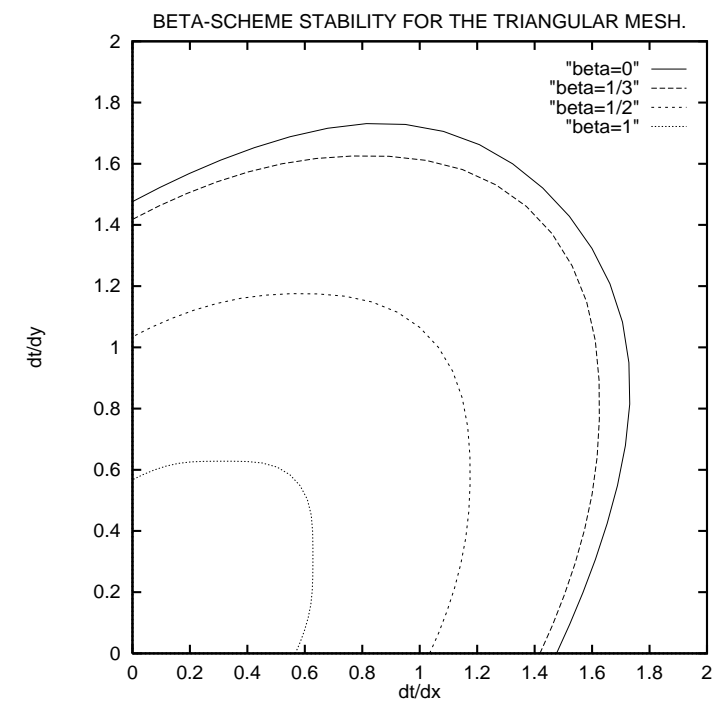

Figure 9. Convection equation.

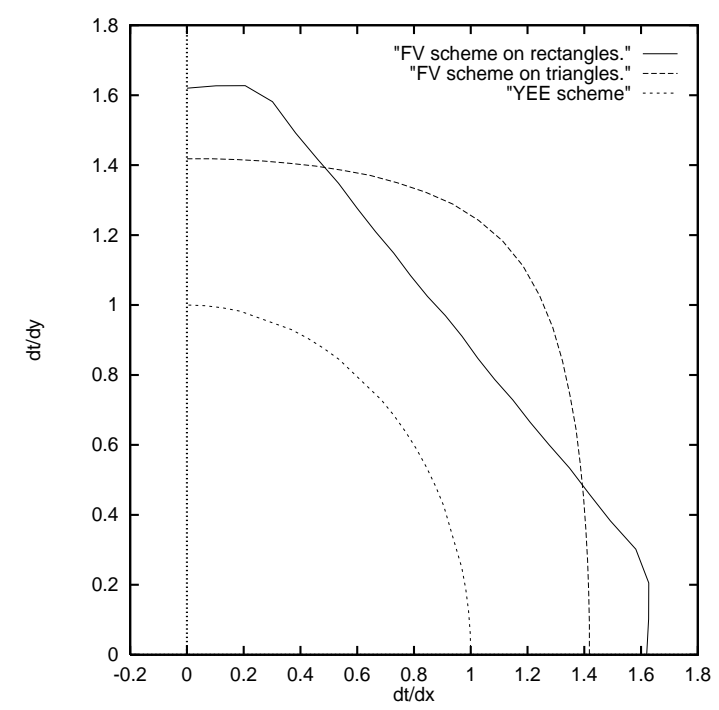

FiguRE 10. Stability domains for Yee and third-order finite volume schemes.

\subsubsection{Comparison with the Yee scheme}

We now compare the stability domains obtained for the Yee scheme and for third-order finite volume schemes applied to the Maxwell system. Figure 10 shows that stability domains are wider for $\beta$-schemes $(\beta=1 / 3)$, on both rectangular and triangular meshes.

On the contrary to the Yee scheme, the finite volume approach has the advantage to extend easily first-order upwind schemes to $\beta$-schemes, and then to achieve high-order accuracy in time and space. This method is also more flexible, as it can be applied to many sorts of meshes. However, $\beta$-schemes require an additional CPU time cost compared to the use of the Yee scheme. 


\section{CONCLUSION}

A stability study concerning a class of finite volume schemes applied to the two-dimensional Maxwell system has been presented here. We have proposed a demonstration of a necessary and sufficient stability condition for the first-order upwind scheme, on a rectangular mesh. In this case, we find the same stability condition for the Maxwell system and for the convection equation: $u_{t}+u_{x}+u_{y}=0$.

High-order finite volume schemes are achieved by using a MUSCL formulation, and stability domains are computed on both triangular and rectangular meshes. We also compare the stability limits obtained for the Maxwell system with those obtained when considering the convection equation. We can observe that the stability domains are wider for the convection equation when using a triangular mesh. On the other hand, stability limits are higher for $\beta$-schemes applied to the Maxwell system on rectangular meshes.

We can note that stability limits vary in the same way with $\beta$ for the Maxwell system and for the convection equation: the limit on the time-step is the highest for a fully-centered scheme. When one of the mesh-step tends to infinity, we find again in both cases (the Maxwell system and the convection equation) the one-dimensional stability condition.

A comparison between the Yee scheme and the finite volume approximation is also proposed. In the case of third-order finite volume schemes, stability domains are wider, on both rectangular and triangular meshes.

The author wishes to thank Serge Piperno, Bernard Larrouturou, Loula Fézoui, Didier Issautier (CERMICS-INRIA Sophia-Antipolis) and J.A. Désidéri (INRIA Sophia-Antipolis) for their fruitful help concerning this study.

\section{REFERENCES}

[1] D.A. Anderson, J.C. Tannehill and R.H. Pletcher, Computational Fluid Mechanics and Heat Transfer, Vol. 1. Hemisphere Publishing Corporation (1984).

[2] J.P. Cioni, Résolution numérique des équations de Maxwell instationnaires par une méthode de volumes finis. Ph.D. thesis, University of Nice - Sophia Antipolis, France (1995).

[3] S. Depeyre and D. Issautier, A new constrained formulation of the Maxwell system. RAIRO Modél. Math. Anal. Numér. 31 (1997) 327-357.

[4] J.A. Désidéri, A. Goudjo and V. Selmin, Third-order numerical schemes for hyperbolic problems. INRIA Report No. 607 (1987).

[5] L. Fezoui, Résolution des équations d'Euler par un schéma de Van Leer en éléments finis. INRIA Report No. 358 (1985).

[6] N. Glinsky, Simulation numérique d'écoulements hypersoniques réactifs hors-équilibre chimique. Ph.D. thesis, University of Nice - Sophia Antipolis, France (1990).

[7] P.D. Lax, A. Harten and B. Van Leer, On upstream differencing and Godunov type schemes for hyperbolic conservation laws. SIAM Rev. 25 (1983) 35-61.

[8] B. Van Leer, Flux vector splitting for the Euler equations. Lect. Notes Phys. 170 (1982) 405-512.

[9] A. Taflove and M.E. Brodwin, Numerical Solution of Steady-State Electromagnetism Scattering Problems Using the TimeDependent Maxwell's Equations. IEEE Trans. Microwave Theory Tech. 23 (1975) 623-630.

[10] K.S. Yee, Numerical solution of initial boundary value problems involving Maxwell's equations in isotropic media. IEEE Trans. Antennas Propag. 14 (1993) 302-307. 